\title{
Competition Regulates the Efficacy of an Identified Synapse in Crickets
}

\author{
D. Shepherd and R. K. Murphey \\ Neurobiology Research Center, State University of New York at Albany, Albany, New York 12222
}

\begin{abstract}
The efficacy of the synaptic contact between an identified sensory neuron and an identified interneuron in crickets is increased when neighboring afferent synapses are removed early in postembryonic life. The physiological changes are correlated with changes in the structure of the presynaptic neuron's axonal arborizations: When neighboring axons are destroyed, there is a shift of the remaining axonal arbors into deafferented regions and an increase in the number of putative contacts with the postsynaptic neuron. Changes in the structure of the presynaptic neuron also directly affect the probability of formation of this synaptic connection. The connection was found in $67 \%$ of the control specimens, but it was present in $100 \%$ of the partially deafferented specimens. The results demonstrate that interactions between growing sensory neurons can influence both the probability of synapse formation and the strength of those connections. This is the first case in which the effects of competition on the structure of a single, identified, presynaptic neuron can be directly related to its synaptic efficacy.
\end{abstract}

Normal development of the nervous system requires not only that neurons make synaptic contact with the correct partners, but also that the strength of the synaptic input be adjusted to particular levels. One mechanism that is believed to regulate the number and efficacy of synaptic contacts is competition. The original idca, and much of the cvidence for competitive interactions, come from studies of afferent projections in the visual system of vertebrates (Hubel et al., 1977; Schmidt et al., 1978; Tieman, 1984; Wiesel and Hubel, 1965). A direct demonstration, however, of the effects of competition on individual central synapses is difficult to obtain. The reason for this is numerical: In most vertebrate sensory systems there are large numbers of pre- and postsynaptic neurons, so one can never study the same synaptic connection under a variety of experimental conditions, and most studies have resorted to statistical sampling. In invertebrates, however, the numerical problems are reduced and it is possible to study identified central synapses physiologically. Here, we describe how competition influences the synaptic connection between an identified presynaptic neuron and an identified postsynaptic neuron in the CNS of the cricket. In brief, we demonstrate that synaptic efficacy, at a particular set of synaptic connections, is modulated by competition and that this is correlated with a change in the number of contacts made by the presynaptic neuron on the postsynaptic neuron.

\footnotetext{
Received Dec. 9, 1985; revised May 7, 1986; accepted May 15, 1986.

This work was supported by NSF Grant RNS-8418797. We wish to thank Drs. J. T. Schmidt, S. B. Tieman, and G. Kamper for reading an early draft of the manuscript. Robert Zucker, Eric Frank, and an anonymous reviewer introduced us to the subtleties of quantal analysis; we take credit for any errors that remain

Correspondence should be addressed to Dr. D. Shepherd, Department of Biological Sciences, State University of New York at Albany, 1400 Washington Avenue, Albany, NY 12222.

Copyright (C) 1986 Society for Neuroscience $0270-6474 / 86 / 113152-09 \$ 02.00 / 0$
}

The cricket cercal sensory system is similar in many respects to sensory systems in a wide variety of other animals. A population of wind-sensitive sensory neurons projects in an orderly manner to the $\mathrm{CNS}$, where connections are made with a variety of postsynaptic cells (Bacon and Murphey, 1984; Murphey, 1981; Murphey et al., 1980; Sakaguchi and Murphey, 1983). But unlike vertebrate sensory systems, each wind-sensitive sensory neuron of the cricket cercal system can be individually identified by the location of the external receptor structures that it uniquely innervates, and can be reliably stained in its entirety by simple methods (Bacon and Murphey, 1984).

In many sensory systems the connectivity is flexible and can adjust to changes in the number of pre- or postsynaptic neurons without complete disruption of function (Palka, 1984; Schmidt et al., 1978; Wiesel and Hubel, 1965). Insects are not an exception, and one form of plasticity in the cricket cercal system is revealed when half of the sensory neurons are deleted by removal of 1 cercus. Normally, the medial giant interneuron (MGI) responds primarily to sensory neurons on the cercus ipsilateral to its dendritic field. When this dominant cercus is removed early in postembryonic life, the MGI becomes more responsive than normal to the remaining contralateral cercus, implying a reorganization of connections (Murphey and Levine, 1980; Palka and Edwards, 1974).

Most of the sensory neurons that are associated with windsensitive receptors do not cross the midline and have axonal arbors restricted to the 1 side of the terminal abdominal ganglion (Bacon and Murphey, 1984), although a few sensory neurons do arborize in both the sides of the CNS. When the left cercus is removed during a juvenilc stagc, a bilatcrally projecting neuron on the right cercus shifts most of its axonal arbor into the deafferented left hemiganglion. It has been suggested that this may account for the changes in the responsiveness of MGI (Murphey and Lemere, 1984). We can now demonstrate that these changes in the structure of the identified presynaptic neuron produce concomitant changes in the strength of the synaptic contacts it makes with the bilaterally homologous MGIs.

\section{Materials and Methods}

\section{Rearing}

Animals were reared as previously described (Matsumoto and Murphey, 1977). Groups of 50-100 animals were treated by removing a cercus during the third larval instar. Each animal was inspected every few days for indications of cercal regeneration, and regenerating cercal buds were removed (Palka and Edwards, 1974).

\section{Staining}

Adult female crickets (Acheta domesticus), 12-24 hr after the last moult, were anesthetized on ice and mounted on wax blocks. The afferent projections of the individual $X$ neurons were stained by placing the broken-off tip of a micropipette, filled with $200 \mathrm{~mm}$ cobalt chloride, over the cut end of the hair. Preparations were placed in a humid chamber for $12 \mathrm{hr}$ at $5^{\circ} \mathrm{C}$. The processing of the tissue and its whole- 
Table 1. Number of varicosities of the 3 identified right $X$ neurons $(X, p X, v p X)$

\begin{tabular}{|c|c|c|c|c|c|c|c|c|c|c|}
\hline \multirow[b]{2}{*}{ Neuron } & \multicolumn{5}{|c|}{ Control } & \multicolumn{5}{|c|}{ Experimental } \\
\hline & $n$ & Left & Right & Total & Left/total & $n$ & Left & Kight & Total & Left/total \\
\hline $\mathrm{X}$ & 10 & $137 \pm 43$ & $202 \pm 40$ & $338 \pm 61$ & $0.40 \pm 0.09$ & 11 & $274 \pm 34$ & $48 \pm 23$ & $322 \pm 45$ & $0.85 \pm 0.06$ \\
\hline $\mathrm{pX}$ & 9 & $143 \pm 23$ & $176 \pm 30$ & $320 \pm 35$ & $0.45 \pm 0.07$ & 7 & $283 \pm 62$ & $60 \pm 27$ & $343 \pm 69$ & $0.83 \pm 0.07$ \\
\hline $\mathrm{vpX}$ & 7 & $126 \pm 24$ & $198 \pm 33$ & $324 \pm 21$ & $0.39 \pm 0.08$ & 10 & $255 \pm 64$ & $85 \pm 48$ & $341 \pm 97$ & $0.75 \pm 0.09$ \\
\hline
\end{tabular}

Means \pm SD are represented. Left and right refer to the location of varicosities with respect to the midline.

mount intensification have been described elsewhere (Johnson and Murphey, 1985). All quantification of the size and distribution of the stained axonal arbors was done as described by Murphey et al. (1983) and Murphey (in press).

To produce simultaneous staining of an afferent arbor and the MGIs, the sensory neuron was first cobalt stained for $6 \mathrm{hr}$ as described above. Either 1 of the 2 MGIs was then stained by iontophoretic injection of a freshly made $100 \mathrm{~mm}$ solution of hexamino-cobalt chloride into the cell soma. Electrode tip resistances were 25-30 M when filled with 2 M potassium acetate; cobalt-containing electrodes had resistances approximately double this value. Injection of 5-10 nA of depolarizing current in $500 \mathrm{msec}$ pulses at $1 \mathrm{~Hz}$ for $1-5 \mathrm{~min}$ provided a level of staining that matched the density of the afferent arbor. Preparations were left for $2-5 \mathrm{hr}$ at $5^{\circ} \mathrm{C}$ before processing to allow diffusion within the interneuron.

\section{Physiological methods}

Adult female crickets between 6 and $24 \mathrm{hr}$ after the final moult were anesthetized on ice and mounted dorsal side uppermost on a wax block. The animal was dissected to expose the ventral nerve cord, and an insulated metal spoon was inserted beneath the terminal abdominal ganglion for support. Intracellular recordings were made from the soma of MGI, with electrodes filled with $2 \mathrm{~m}$ potassium acetate and having resistances of 25-30 M .

The activity of the sensory neuron was recorded extracellularly, using methods similar to those used by Bacon and Murphey (1984). The required sensory hair was cut, and a saline-filled micropipette was placed over it. Using this arrangement, which is shown schematically in the inset to Figure 2, the spontaneous activity of the sensory neuron could be recorded and its frequency controlled by passing current through the electrode. To prevent habituation of the synapse during experiments, $1-4 \mathrm{nA}$ of hyperpolarizing current was passed through the electrode in order to maintain the spike frequency of the sensory neuron at less than $0.1 \mathrm{~Hz}$. Signal averaging was performed using a Nicolet 4074 digital oscilloscope, triggered by the presynaptic spike.

All experiments were performed at a temperature of $20-22^{\circ} \mathrm{C}$. Higher temperatures increased transmitter release by the sensory neuron, making quantal analysis difficult.

\section{Quantal analysis}

Individual EPSP amplitudes were measured, stored, and analyzed online using an IBM PC personal computer and self-written software. We sampled and stored the size of as many EPSPs as each preparation permitted, with a minimum acceptable sample size of 50 .

At the cricket vpX-neuron to MGI synapse, as well as at other central synapses, spontaneous miniature EPSPs cannot be used to determine the unit quantal size $(q)$ and thereby calculate the mean quantal content $(m)$. On the other hand, it is possible, using less direct methods, to obtain estimates of $m$ and $q$. We have estimated $m$ and $q$ in 2 ways.

\section{Failure analysis}

It is possible to obtain an estimate of $m$ by examining the ratio of failures $\left(n_{0}\right)$ to the total number of trials $(N)$, where, assuming that the number of quanta released follows a Poisson distribution, $m=\ln N / n_{0}$ and $q=V / m$ (Baxter et al., 1985; Martin, 1966). The number of failures was determined by observation during experiments. The standard error of $m[\mathrm{SE}(m)]$ as determined by failure analysis is calculated from

$$
\mathrm{SE}(m)=1-P_{0} / N P_{0}
$$

where $P_{0}=n_{0} / N$ (Martin, 1966).

\section{Coefficient of variation}

Assuming that the number of quanta released at each stimulation is binomially distributed, the coefficient of variation of the evoked EPSPs could be used to estimate $m$. The average probability of the release of a quantal unit $(p)$ is calculated from the expression $p=V / V_{\max }$, where $V$ is the mean amplitude of the evoked EPSP, and $V_{\max }$ is the amplitude of the largest EPSP in the sample; from $p$, we could calculate $m ; m=$ $1-p / \mathrm{CV}^{2}$, where $\mathrm{CV}$ is the coefficient of variation of the sample. The amplitude of a unit quantal depolarization $(q)$ could be calculated from $q=V / m$ (Grantyn et al., 1984).

\section{Results}

\section{Anatomy of a connection}

Three sensory neurons possessing bilateral axonal arborization have been identified, and each is associated with a different receptor hair located on the medial aspect of the cercus (Figs. $1 A$ and 2, inset). The receptors, and their associated neurons, have becn named according to their position along the cercus; the most distal is called $\mathrm{X}$, the next more proximal is called proximal $X(p X)$, and the most proximal, very proximal $X$ (vpX). These are the 3 most proximal neurons with bilaterally projecting axons. There are others located more distally, but in total their numbers are small: We estimate that fewer than 25 out of a total of 1000 neurons on a cercus project bilaterally. Cobalt staining of the individual $X$ neurons on the right cercus of normal adult crickets shows that apart from the location of their somata in the periphery, they are indistinguishable at the light-microscopic level. The soma of each of the $X$ neurons projects an axon through the cercal nerve into the terminal abdominal ganglion, each axon crosses the midline and arborizes in bilaterally homologous regions of neuropil (Figs. $1 A$ and 2 , inset). The size of the arbor, as measured by the number of varicosities in it, is similar for each of the $X$ neurons, as is the bilateral distribution of the varicosities, with $60 \%$ of the varicosities located ipsilateral to the soma (Table 1).

When the left cercus was removed during a juvenile stage, and the $\mathrm{X}$ neurons on the remaining cercus examined in the adult, the structure of the $X$ neurons was distorted. There was an increased number of varicosities in the denervated, left half of the ganglion and a decreased number in the right half of the ganglion. Murphey and Lemere (1984) first demonstrated this for the neuron $X$, and Table 1 shows that the same was true for all 3 identified $X$ neurons. They shift most of their arbors $(80 \%$ of their varicosities) into the left side of the nervous system, and the total number of varicosities remains constant.

These anatomical changes in the terminal arbors imply changes in the connections made by the $\mathrm{X}$ neurons with their postsynaptic targets. In the region where the $\mathrm{X}$ neurons expand, we would expect them to make more contacts with postsynaptic cells; and in the region where the $X$ neurons retract, we would expect a loss of synaptic contact. We have assessed this idea in a preliminary way by counting the number of putative contacts between the $\mathrm{X}$ neurons and the MGIs, visible at the light-microscopic level. Examination of whole-mounted preparations, 

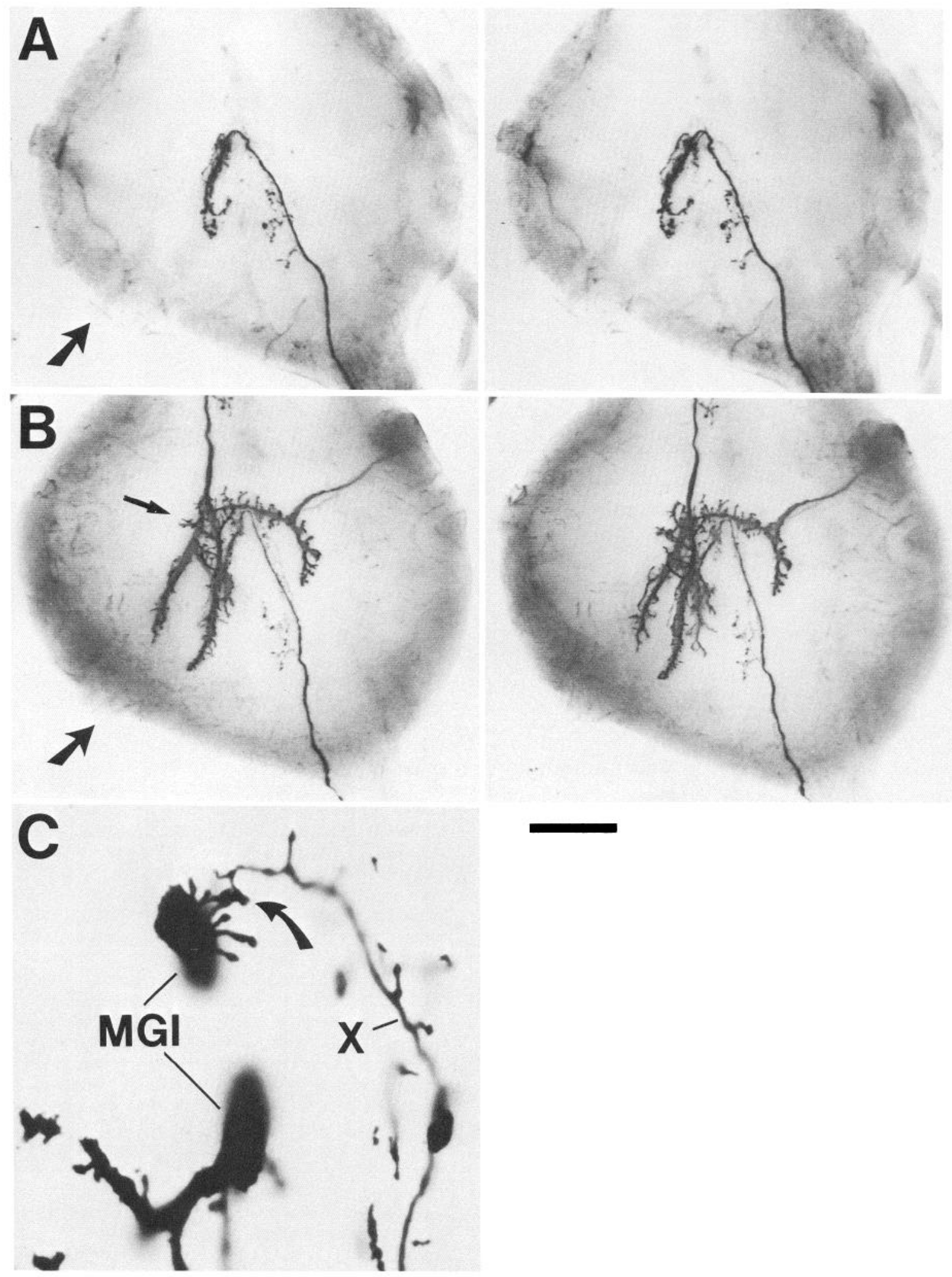

Figure 1. Structure of the identified pre- and postsynaptic neurons. A, Stereophotographs of the axonal arborization of the right pX in a specimen reared without the left cercus (the arrow indicates the missing cercal nerve). Note that most of the varicosities are located to the left of the midline. $B$, Stereophotographs of a preparation reared without the left cercus in which the right pX and the left MGI were stained. By comparing $A$ and $B$ it can be seen that the afferent and the interneuron are likely to contact one another. $C$, Cross section of the specimen shown in $B$ (plane of section indicated by small arrow), illustrating a putative contact between the pX neuron and the MGI (arrow). The midline is at the right edge of the photograph. Note the many processes that do not touch the MGI. Calibration bar: $A$ and $B, 120 \mu \mathrm{m} ; C, 17 \mu \mathrm{m}$. 




Figure 2. Simultaneous recordings from identified sensory neurons and an identified interneuron. The recording situation is shown in the schematic at the left. The lower trace in each recording is the action potential in the sensory neuron recorded extracellularly from the hair. The upper trace in each pair is the EPSP recorded in the MGI. Each pair represents the average of 30 trials. The recordings were selected to represent the average size of the EPSP in 5 specimens. Note that there is a clear EPSP when vpX is active, a smaller EPSP when pX is active, and no response when X is active.

in which an $\mathrm{X}$ neuron and one MGI were stained with cobalt, demonstrated close association between the $X$ neuron's axonal arbor and the dendrites of the MGIs (Fig. $1 B$ ).

These preparations were sectioned in the transverse plane and the sections examined for putative synaptic contacts. A single contact was scored when the stained profiles of the 2 neurons became indistinguishably close (e.g., Fig. $1 C$ ). We obtained the largest number of examples of such preparations when $\mathrm{pX}$ and MGI were simultaneously stained. The data show that, following the reorganization of the $\mathrm{X}$ neuron arbor, there was a change in the number of putative contacts between the $X$ neuron and both right and left MGIs (Table 3 ). In the case of the right $p X$ to-left MGI connection the number of putative contacts increased from 5.5 to 14 , whereas with pX-to-right MGI the number of putative contacts decreased from 10 to 3.5 . Although this light-microscopic analysis does prove that the number of contacts has changed, there is a very strong correlation between the anatomical observations and the physiological results. In particular, the number of contacts parallels the observed increases in synaptic efficacy (see next section and Table 3 ).

\section{Physiology of the synapse}

To confirm that a synaptic contact existed between the $\mathrm{X}$ neurons and the MGIs, we recorded simultaneously from 1 of the sensory neurons and either 1 or the other of the MGIs. In general, action potentials in the most proximal of the $X$ neurons (vpX) were followed by small-amplitude $(0.2-1.0 \mathrm{mV})$, shortlatency (2.4-3.4 msec) EPSPs in both left and right MGIs (Figs. 2 and $3 A$ ). This latency and the conduction distances suggested a synaptic delay of less than $1 \mathrm{msec}$, which is consistent with other examples of monosynaptic connections in insects (Burrows, 1975; Callec et al., 1971; Pearson et al., 1976). Although there is no absolute anatomical proof that this connection is monosynaptic, there is circumstantial evidence in support of the idea. Electron-microscopic studies of degeneration have shown that some cercal afferents do end on the giant fibers (Palka and Edwards, 1974; R. K. Murphey, unpublished observations). Furthermore, recent evidence has made it clear that these af- ferent contacts come from filiform receptors and not from the other types of receptors such as appressed hairs (Murphey, 1985).

Similar recordings made using the more distal X neurons ( $\mathrm{pX}$ or $\mathrm{X}$ ) revealed that their contacts were considerably weaker than that of $\mathrm{vpX}$, as shown for the left MGI in Figure 2. Neuron $X$ never produced a detectable EPSP in either MGI, and pX evoked potentials of $0-0.2 \mathrm{mV}$. These differences in the strength of the synaptic contact are correlated with 2 parameters: (1) the position of the $\mathrm{X}$ neuron (the more distally located the sensory neuron, the weaker the contact) and (2) the age of the neuron. Neuron $\mathrm{X}$ is the oldest, $\mathrm{pX}$ the next oldest, and vpX the youngest (Murphey, in press). Whether these correlations are causally related to synaptic strength is currently under investigation. The experiments described below were performed using the 2 most proximal neurons, $\mathrm{pX}$ and $\mathrm{vpX}$.

\section{Probability of synaptic contact}

Recordings made from the vpX neuron and the 2 postsynaptic cells in different animals revealed that the vpX neuron did not always form a detectable synaptic contact. In untreated adult crickets, the right vpX neuron formed a physiologically detectable synapse with the left MGI in $67 \%$ of the specimens and with the right MGI in 78\% of the specimens (Table 2). In animals lacking a left cercus, the probability of recording a detectable synapse increased to $100 \%$ for the left MGI and dccrcascd to zero for the right MGI. Thus, connectivity appears to be probabilistic and is correlated with the observed changes in the structure of the presynaptic terminal; that is, when the axonal arborization shrinks, the probability of a detectable synapse decreases; and when the axonal arborization expands, the probability of a detectable contact increases.

\section{Competition changes synaptic strength}

In untreated adult crickets, action potentials in the vpX neuron of the right cercus produced short-latency EPSPs in both the left and right MGIs. Figure $3 A$ shows signal-averaged EPSPs obtained by triggering on the vpX impulses and averaging 30 

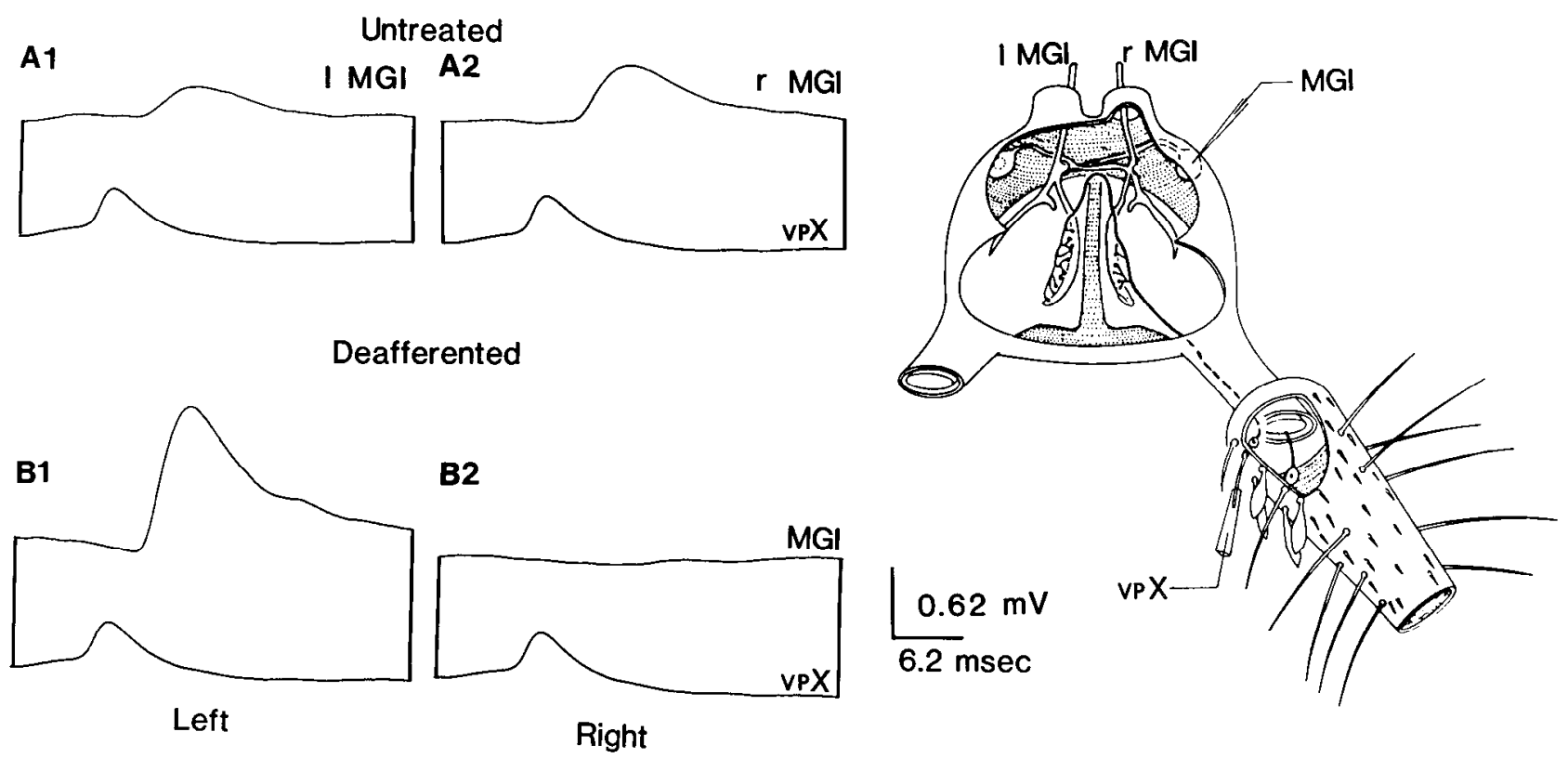

Figure 3. Signal-averaged (30 sweeps) EPSPs recorded in the soma of the bilaterally homologous medial giant interneurons evoked by action potentials in the right vpX neuron. $A$, Examples recorded from the left $(A I)$ and right $(A 2)$ MGI of normal animals. $B$, Examples recorded from the left $(B 1)$ and right $(B 2)$ MGI of animals reared without a left cercus. These examples were chosen because they are representative of the mean EPSP amplitudes obtained from 5 control specimens and 5 treated specimens in which we successfully recorded from vpX and both MGIs.

responses recorded from first the left and then the right MGI of a specimen. The examples were chosen to represent the mean EPSP sizes obtained from 5 preparations (Table 2). In general, the right vpX evoked a larger EPSP in the right MGI $(0.43 \mathrm{mV}$; Fig. 3A2) than in the left MGI $(0.25 \mathrm{mV}$; Fig. 3A1). These physiological results were consistent with the distribution of the varicosities of the vpX neurons; $60 \%$ were located near the right MGI, $40 \%$ near the left MGI.

In experimental specimens, action potentials in the right $\mathrm{vpX}$ elicited EPSPs only in the left MGI (Fig. 3B1); no EPSPs were detected in the right MGI (Fig. 3B2). The EPSP in the left MGI was clearly larger in the deafferented crickets (Fig. $3 B 1$ ) than in the normal adult crickets (Fig. 3A1). Again, the examples were chosen to represent the mean EPSP sizes obtained from 5 preparations in which we successfully recorded from both right and left MGI (Table 2). These physiological changes were consistent with the anatomical changes produced by chronic removal of 1 cercus. On the left, denervated, side where the right vpX neuron expanded its arbor, there was an apparent increase in the strength of contact with the left MGI. On the right side, where $\mathrm{vpX}$ retracted its arbor, there was a decrease in the strength of contact with the right MGI.

\section{Variation in EPSP size}

The individual EPSPs evoked by the $\mathrm{vpX}$ neuron normally fluctuate in size, as shown in Figure 4, and representative histograms of the variation are shown for treated and control specimens in Figure 5. It seemed likely to us that transmission at this synapse is quantal in nature, as at most other chemical synapses. The best evidence for this comes from examination of the histograms of EPSP size in experimental animals, in which conditions are the most favorable for recording the unit event and thus the histograms are presumably the most accurate. (Two examples are illustrated in Fig. 5, B, C). The histograms are multimodal and theoretical discrete distributions can bc found that approximate the observed distributions. It is difficult to prove the quantal hypothesis because miniature end-plate potentials, which can serve as independent measures of the size of the quantal event, cannot be distinguished from spontaneous synaptic potentials due to the many other synapses ending on these central neurons. Assuming transmission is quantal, then the frequency histograms of EPSP amplitudes should provide further clues to the mechanism of the observed changes in synaptic efficacy.

Table 2. Specificity and efficacy of the synaptic connection between the right vpX and the right and left MGI

\begin{tabular}{|c|c|c|c|c|c|c|}
\hline \multirow[b]{2}{*}{ Group } & \multicolumn{3}{|c|}{ Right vpX/LMGI } & \multicolumn{3}{|c|}{ Right $v p X / R M G I$} \\
\hline & $\begin{array}{l}\text { Speci- } \\
\text { mens } \\
\text { tested }(n)\end{array}$ & $\begin{array}{l}\% \text { detect- } \\
\text { able } \\
\text { synapses }\end{array}$ & $\begin{array}{l}\text { Mean EPSP } \\
\text { size }^{a} \\
(\mathrm{mV} \pm \mathrm{SD})\end{array}$ & $\begin{array}{l}\text { Speci- } \\
\text { mens } \\
\text { tested }(n)\end{array}$ & $\begin{array}{l}\% \text { detect- } \\
\text { ahle } \\
\text { synapses }\end{array}$ & $\begin{array}{l}\text { Mean EPSP } \\
\text { size }^{a} \\
(\mathrm{mV} \pm \mathrm{SD})\end{array}$ \\
\hline Control & 12 & 66.6 & $0.25 \pm 0.23$ & 9 & 77.7 & $0.43 \pm 0.32$ \\
\hline Experimental & 12 & 100.0 & $1.27 \pm 0.35$ & 10 & 0.0 & 0.00 \\
\hline
\end{tabular}

${ }^{a}$ Mean EPSP size is calculated only from 5 preparations in which paired recordings from both left and right MGIs were obtained in each specimen. 

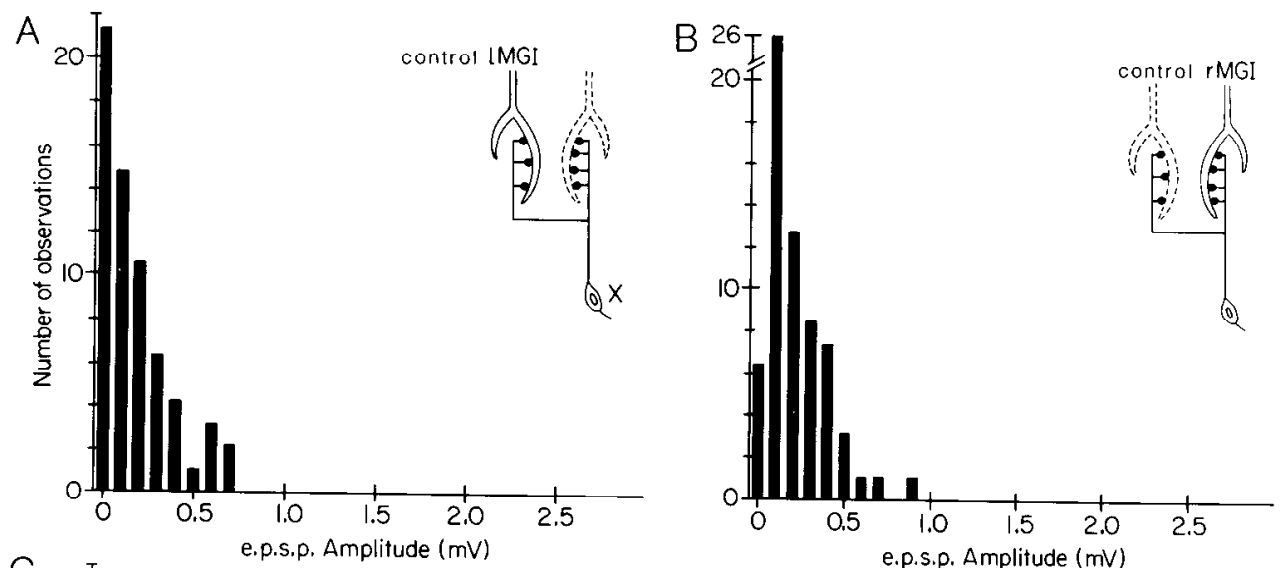

Figure 4. Effects of the reorganization of the vpX axonal arbor on EPSP amplitude fluctuation. $A$ and $B$, Amplitude histograms of EPSPs recorded from the left $(A)$ and right $(B)$ MGI of 2 control specimens in response to action potentials in vpX. $C$, Amplitude histogram for the left MGI of an experimental specimen. The inset in each histogram schematizes the recording situation. The afferent is the

C

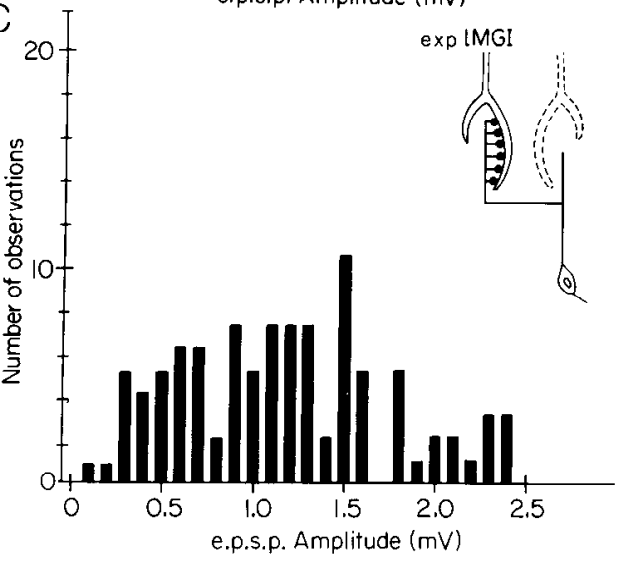

D

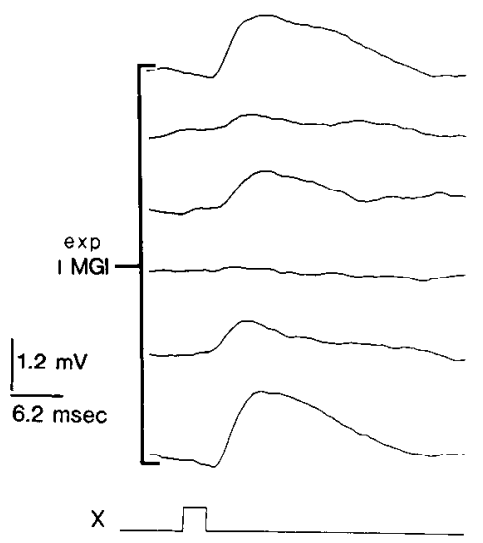
right vpX neuron, and the dendrites of both MGIs are outlined. The recorded neuron is shown as a solid outline and the bilateral homolog as a dashed outline. The number of afferent contacts with MGI is drawn to illustrate the postulated changes in connections. $D$, Sample recordings from the left MGI of an experimental specimen showing the range of variability of EPSP sizes. The action potential of the $\mathrm{vpX}$ neuron was used to trigger the sweep, and a digital representation of the trigger is shown on the lower trace. Each of the 6 recordings from this MGI was aligned with the trigger event.

In particular, we hoped to be able to distinguish pre- from postsynaptic changes. We focused our attention on the left MGI. Since the presynaptic neuron shifts arbor into the region near the left MGI, it seemed likely that this would contribute to the enhanced synaptic strength. The histograms in Figure 5 show that there were many more failures of transmission in the control left MGI than in the deafferented left MGI. This suggested that mean quantal content had increased, which is diagnostic of a presynaptic change.

A number of methods are available for determining the mean quantal content $(\mathrm{m})$ at a synapse. The direct method is to determine the ratio of the average EPSP size to the average spontaneous miniature end-plate potential for that synapse. However, as pointed out above, this method cannot be applied because at these central synapses one cannot identify the spontaneous miniature potentials. A second method, the failures method, depends on the assumption that the distribution is Poisson and that a failure of transmission can be reliably distinguished from release of one or more quanta, in which case $m=\ln$ (trials/ failures). The failure method when applied to the control MGIs provided $m$ values betwecn 1.14 and 1.6 (Table 3 ). In experimental left MGIs, the synaptic input was enhanced such that there were very few failures, and $m$ values ranged from 3.3 to 4.6 , when they could be calculated (Table 3 ). Although estimates of $m$ by the failure method are certainly less reliable in experimental animals than in controls, it appears that $m$ has increased.

A less direct method for estimating $m$, the binomial method, had the advantage that it did not depend upon failures and thus could be applied equally well to both deafferented and normal MGIs. It had the disadvantage that a variety of assumptions are implicit in the method, and we could not establish the validity of these assumptions (Baxter et al., 1985; Zucker, 1977). For example, determination of the variation of the quantal event was not possible because we could not observe spontaneous miniatures and therefore could not determine its variance. With these cautions in mind, we applied this method to obtain estimates of $m$. The trends were the same as with the failure method; for the left MGI of control specimens, $m$ was between 0.67 and 0.96 ; for deafferented animals, $m$ ranged from 2.28 to 3.05 . Thus, analysis of the fluctuations in EPSP size supports the idea that changes in the presynaptic cell, reflected in these estimates of $m$, contribute to the observed changes in synaptic efficacy. In particular, the shift of presynaptic arbors to the left side of the nervous system correlates with increases in the strength of the synapse.

These analyses were limited by features of the experiment that could not be controlled. The most important limitation was that we could not obtain very large numbers of EPSPs. There were 3 reasons for this: (1) The synapse is labile, habituating at frequencies as low as $1 \mathrm{~Hz}$. Thus, the frequency of presynaptic action potentials must be kept at very low levels (we kept the frequency below $0.1 \mathrm{~Hz}$ ). (2) Although we could hyperpolarize the presynaptic cell, we could not stimulate the presynaptic cell on command. We had to depend on spontaneous activity to collect EPSPs. (3) We were able to hold the recordings only for limited amounts of time. In practice, this meant that we could not obtain more than about 100 events from a single recording. In spite of these limitations, we concluded that at least part of the obscrved change in synaptic strength could be attributed to changes in the presynaptic cell and were correlated with changes in its anatomy.

Changes in the postsynaptic cell may have also contributed to the larger EPSP in the left MGI of experimental animals. The deafferented dendrites of the left MGI were smaller in caliber and length than in controls, and these factors may have increased the input resistance. Based on these morphological changes, Levine (1978) calculated that the input resistance near a synapse 

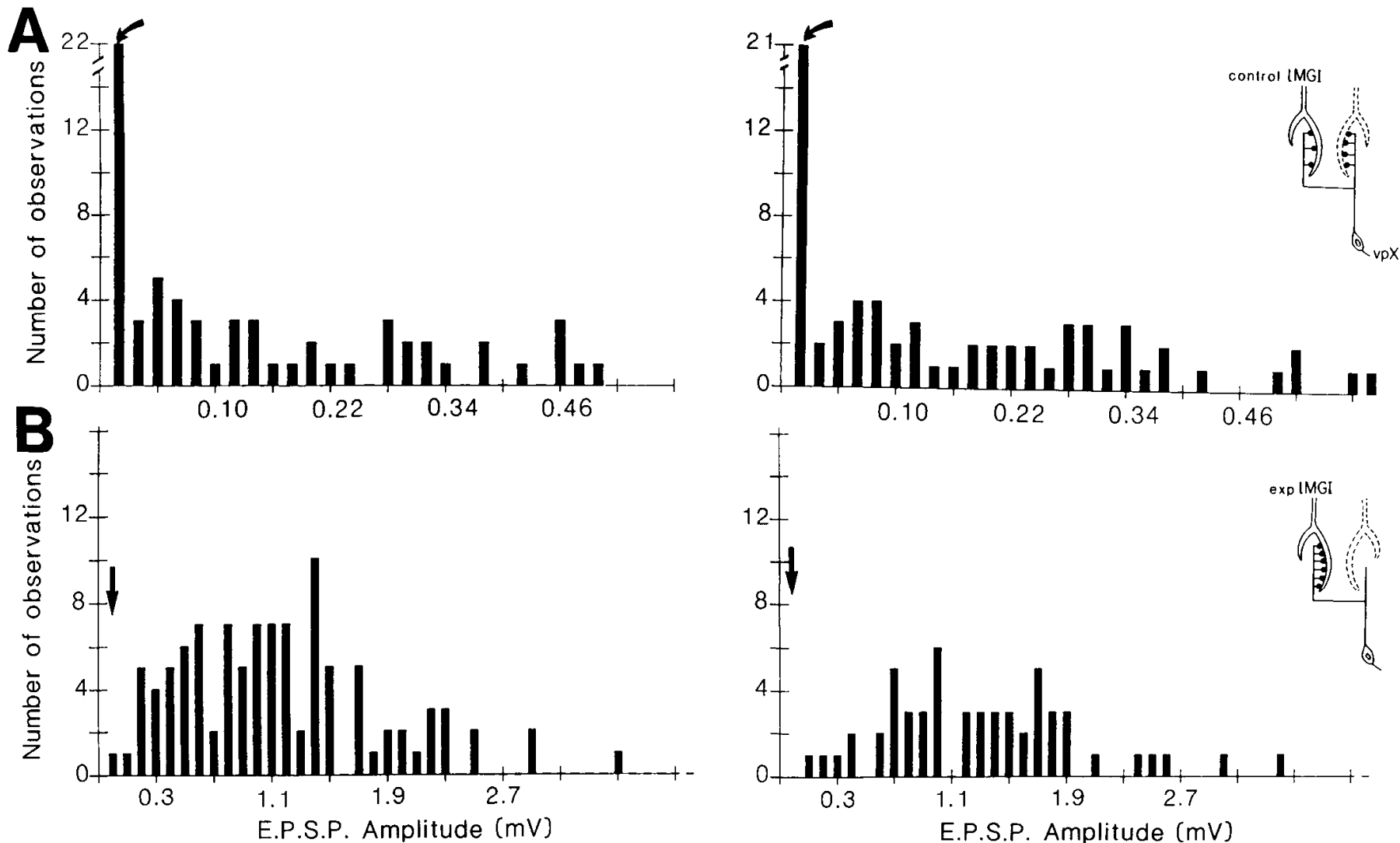

Figure 5. Changes in the amplitude histograms for the left MGI in control and treated specimens. $A$, Histograms for 2 control specimens (preparations 3 and 2 of Table 3). B, Histograms for 2 experimental specimens (preparations 9 and 8, Table 3). The solid arrows indicate failures, based on judgments made during the experiment. The insets at the right schematize the recording situation. Note the differences in scale on the $x$ axis.

would be increased approximately 3 times. An increase of this magnitude is unlikely to account for the observed changes since the average amplitude increased 2-7 times. Thus, changes in both the pre- and postsynaptic cell may have contributed to the changes in synaptic efficacy.

\section{Discussion}

One of the unique advantages of the preparation used in this study is that it is possible to investigate, electrophysiologically, the synaptic connection between homologous pre- and postsynaptic neurons in different animals under different experimental conditions. The results obtained provide several important insights into the mechanisms underlying synaptogenesis and the regulation of synaptic efficacy.

\section{Specificity of the synaptic connection between $v p X$ and MGI}

One interesting feature of this study is that the presynaptic neuron did not always form a detectable synaptic contact with the 2 MGIs. Since this particular synaptic connection was absent in some specimens, neither the pre- nor the postsynaptic cell depended upon a particular partner for survival. In normal animals, the right $\mathrm{vpX}$ neuron made a functional contact with the right MGI in 78\% of the animals and with the left MGI in 67\% of the animals. This suggested that the identity of the sensory neuron did not specify the exact connections made by that cell, but rather dictated the cell's growth to a particular local neuropil region. Apparently, once the axon of the sensory neuron reaches the correct local region, connectivity with a particular dendrite is stochastic, and local interactions with other sensory neurons can influence the connections made.

Similar variability in synaptic connections has also been described for the synaptic contact between the descending contra- lateral movement detector and the fast extensor tibiae motoneuron of the locust, in which it was shown that the presence or absence of a synaptic connection was associated with differences in the anatomy of the 2 neurons (Pearson and Goodman, 1979). The implication of this finding is that synaptic connections between neurons in invertebrates are not as rigidly determined as is generally believed (Easter et al., 1985).

In Ireated animals the probabilities of the right vpX contacting MGI were changed to 0\% for the right MGI and 100\% for the left MGI. Apparently competition alters the probability of connections. In the region where $\mathrm{vpX}$ withdraws varicosities, it loses contact with the MGI; in the region where it adds varicosities, it adds synaptic contacts with the MGI.

\section{Synaptic efficacy}

Removal of 1 cercus early in life increased the responsiveness of the deafferented MGI to stimulation of the afferents on the remaining cercus (Murphey and Levine, 1980). These changes in synaptic input to the MGIs could have been due to any of several factors: changes in the number of presynaptic contacts, changes in the efficacy of existing contacts, changes in the number of postsynaptic receptors, or a change in the input resistance of the MGI.

A number of results argue that part of the change can be attributed to changes in the presynaptic cell. First, the lack of a detectable EPSP between the right $\mathrm{vpX}$ and the right MGI was strong physiological support for the idea that the presynaptic cell had reorganized its synaptic contacts, indicating that the sensory neuron had withdrawn its synaptic connections with the right MGI. This conclusion is buttressed by the anatomical data showing a loss of varicosities and a loss of putative synaptic contacts on the right side of the ganglion. Second, the deaffer- 
ented left MGI received much stronger (2-7 times normal) input from the right $\mathrm{vpX}$ neuron. The results of the quantal analysis (Fig. 5 and Table 3) supported the idea that the presynaptic cell changed in these experiments. The estimates of $m$ for the left MGI of experimental animals were shown to increase, relative to controls, and this is diagnostic of a presynaptic change. The anatomical data (Table 1) also suggest a presynaptic change since the number of contacts between the right $\mathrm{pX}$ and the left MGI of deafferented animals was greater than that observed in controls. Anatomical changes are not, however, a prerequisite for changes in synaptic efficacy. Parnas et al. (1984) have demonstrated in lobster muscle that quantal transmitter release of a single motor neuron was increased following elimination of a second axon innervating the same muscle, without any apparent morphological changes. Evidently, physiological changes in presynaptic neurons can also produce increases in synaptic efficacy without changing the number of synaptic contacts.

There were other changes in the nature of the synaptic contact revealed in the quantal analysis. The depolarization produced by a single quantum of transmitter $(q)$ was larger in the left MGI of deafferented specimens ( $q$; Table 3 ) than it was in the left MGI of control animals. This change in $q$ could have been due to a variety of factors. For example, the dendrites of MGI were known to be stunted by the treatment (Murphey and Levine, 1980), and this would be expected to have increased the input resistance. This hypothesis is difficult to test directly in this system, but calculations based on the changes in anatomy of the postsynaptic cell suggested a 3 -fold increase in the input resistance of the deafferented MGI. A second possibility was a change in the position of the contacts to more favorable sites on the dendrites of MGI. However, examination of the positions of the contacts on the dendrites of the MGIs revealed no differences in the relative positions of the putative synaptic contacts in normal and treated crickets. The increase in the size of the quantal event might influence the statistical analyses by changing our ability to detect the EPSPs. However, it is highly unlikely that the shift in the histograms observed in Figure 5 could be accounted for by this mechanism.

\section{Conservation}

One of the most interesting features of the $\mathrm{X}$ neurons (Murphey and Lcmerc, 1984; Murphcy, in press; and present results) is that the total number of varicosities is intrinsically limited, even when the bilateral distribution is altered experimentally. This result has been interpreted to mean that the arbor size remains constant and that all structural changes occur within these limitations (Murphey and Lemere, 1984). Similar observations have been made for mammalian central neurons (Frost and Schneider, 1979; Schneider, 1973) and amphibian neuromuscular junctions (Angaut-Petit and Mallart, 1979). The present physiological results suggest a similar phenomenon may occur in insects. Increases in $m$ observed in the left MGI are always accompanied by a loss of a connection to the right MGI. Thus, the physiological results parallel the anatomical conservation, suggesting that synaptic strength is also conserved (Table 3).

The conservative nature of the sensory neuron is particularly important to the explanation of the actions of competition. Competition apparently modulates connectivity within certain limits intrinsic to the neuron. Thus, when deafferentation provides attractive sites for synaptogenesis, the $\mathrm{X}$ neurons respond by forming synapses in the newly available space. But, since the $\mathrm{X}$ neurons are limited in size, this means that a new synaptic contact made on 1 target cell must be made at the expense of a synaptic contact on another target neuron.

\section{Conclusion}

To conclude, our results demonstrate 3 important points about synaptogenesis in invertebrates. First, an identified sensory neuron does not always form a detectable synaptic connection with
Table 3. Summary of the results of the quantal analysis of the $\mathrm{vpX}$ to MGI synaptic connection

\begin{tabular}{|c|c|c|c|c|}
\hline \multirow[b]{2}{*}{$\begin{array}{l}\text { Prepa- } \\
\text { ration }\end{array}$} & \multicolumn{2}{|c|}{ Right vpX/LMGI } & \multicolumn{2}{|c|}{ Right vpX/RMGI } \\
\hline & Failures $^{a}$ & $\begin{array}{l}\text { Vari- } \\
\text { ance }\end{array}$ & Failures $^{a}$ & $\begin{array}{l}\text { Vari- } \\
\text { ance }\end{array}$ \\
\hline \multicolumn{5}{|c|}{ Control } \\
\hline$m$ & $1.34 \pm 0.22$ & 0.94 & $1.97 \pm 0.26$ & 1.55 \\
\hline$q$ & 0.12 & 0.26 & 0.09 & 0.17 \\
\hline$m$ & $1.18 \pm 0.18$ & 0.96 & $1.50 \pm 0.28$ & 1.10 \\
\hline$q$ & 0.17 & 0.23 & 0.19 & 0.35 \\
\hline$m$ & $1.14 \pm 0.18$ & 0.67 & $1.59 \pm 0.31$ & 1.13 \\
\hline$q$ & 0.14 & 0.36 & 0.22 & 0.38 \\
\hline Cont & \multicolumn{2}{|c|}{4,7} & \multicolumn{2}{|c|}{10,10} \\
\hline
\end{tabular}

Experimental

$\begin{array}{lll}m & 3.34 \pm 0.57 & 3.05 \\ q & 0.31 & 0.39 \\ m & - & 2.46 \\ q & - & 1.04 \\ m & 4.60 \pm 0.99 & 2.28 \\ q & 0.34 & 0.57\end{array}$

$\operatorname{Contacts}^{b}(n) \quad 14,14$

3,4

Estimates of $m$ and $q$ are given for individual preparations as calculated by the failure analysis and variance.

${ }^{a}$ Estimate of $m \pm \mathrm{SE}(m)$.

${ }^{b}$ The number of contacts between MGI and $\mathrm{pX}$ as determined at the lightmicroscopic level. Absolute value for each specimen.

a particular postsynaptic neuron, and therefore this particular synaptic connection is not rigidly specified. Second, it seems likely that competition regulates synaptic efficacy by determining the number of synaptic contacts formed with a given interneuron. Thus, removal of competing neurons increases the number of contacts that $\mathrm{vpX}$ will make with the left MGI and concomitantly increases the number of quanta released at the synapses with the left MGI. Finally, the reorganization of the axonal arbor of the $\mathrm{X}$ neurons is conservative in the sense that the total size of the axonal arbor is intrinsically detcrmined, and any reorganization occurs within this constraint. The physiological reflection of this is that increases in $m$ observed in the left MGI are accompanied by decreases in $m$ observed for the right $\mathrm{MGI}$.

\section{References}

Angaut-Petit, D., and A. Mallart (1979) Dual innervation of end-plate sites and its consequences for neuromuscular transmission in muscles of adult Xenopus laevis. J. Physiol. (Lond.) 289: 203-218.

Bacon, J. P., and R. K. Murphey (1984) Receptive fields of cricket giant interneurons are related to their dendritic structure. J. Physiol. (Lond.) 352: 601-623.

Baxter, D. A., G. D. Bittner, and T. H. Brown (1985) Quantal mechanism of long term synaptic potentiation. Proc. Natl. Acad. Sci. USA 82: 5978-5982.

Burrows, M. (1975) Monosynaptic connexions between wing stretch receptors and flight motor neurons of the locust. J. Exp. Biol. 62: 189-219.

Callec, J. J., J. C. Guillet, Y. Pichon, and J. Boistel (1971) Further studies on synaptic transmission in insects. II. Relations between sensory information and its synaptic integration at the level of a single giant axon in the cockroach. J. Exp. Biol. 55: 123-149.

Easter, S. S., D. Purves, P. Rakic, and N. C. Spitzer (1985) The changing view of neural specificity. Science $230: 507-511$.

Frost, D. O., and G. E. Schneider (1979) Plasticity of retinofugal projections after partial lesions of the retina in newborn Syrian hamsters. J. Comp. Neurol. 185: 517-568.

Grantyn, R., A. I. Shapovalov, and B. I. Shiriaev (1984) Relation 
between structural and release parameters at the frog sensory-motor synapse. J. Physiol. (Lond.) 349: 459-474.

Hubel, D. H., T. N. Wiesel, and S. LeVay (1977) Plasticity of ocular dominance columns in monkey striate cortex. Philos. Trans. R. Soc. Lond. [Biol.] 278: 377-409.

Johnson, S. E., and R. K. Murphey (1985) The afferent projection of mesothoracic bristle hairs in the cricket, Acheta domesticus. J. Comp. Physiol. 156: 369-379.

Levine, R. B. (1978) Inhibition of sensory interneurons in the cricket Acheta domesticus and its modification during development. Ph.D. thesis, State University of New York, Albany, New York.

Martin, A. R. (1966) Quantal nature of synaptic transmission. Physiol. Rev. 46: 51-66.

Matsumoto, S. G., and R. K. Murphey (1977) Sensory deprivation during development increases the responsiveness of cricket giant interneurons. J. Physiol. (I ond.) 268:533-548.

Murphey, R. K. (1981) The structure and development of a somatopic map in crickets: The cercal afferent projection. Dev. Biol. 88: 236246.

Murphey, R. K. (1985) A second cricket cercal sensory system: Bristle hairs and the interneurons they activate. J. Comp. Physiol. 156: 357367.

Murphey, R. K. (in press) Competition and the dynamics of axon arbor growth in the cricket. J. Comp. Neurol.

Murphey, R. K., and C. A. Lemere (1984) Competition controls the growth of an identified axonal arborization. Science 224: 1352-1355.

Murphey, R. K., and R. B. Levine (1980) Mechanisms responsible for the changes observed in the response properties of partially deafferented insect interneurons. J. Neurophysiol. 43: 367-382.

Murphey, R. K., A. Jacklet, and L. Schuster (1980) A topographic map of sensory cell terminal arborizations in the cricket CNS: Correlation with birthday and position in a sensory array. J. Comp. Neurol. 191: 53-64.

Murphey, R. K., S. E. Johnson, and D. S. Sakaguchi (1983) Anatomy and physiology of supernumerary cercal afferents in crickets: Implications for pattern formation. J. Neurosci. 3: 312-325.
Palka, J. (1984) Precision and plasticity in the insect nervous system. TINS 7: 455-456.

Palka, J., and J. S. Edwards (1974) The cerci and abdominal giant fibres of the house cricket, Acheta domesticus. II. Regeneration and effects of chronic deprivation. Proc. R. Soc. Lond. [Biol.] 185: 105121.

Parnas, I., J. Dudel, I. Cohen, and C. Franke (1984) Strengthening of synaptic contacts of an excitatory axon on elimination of a second excitatory axon innervating the same target. J. Neurosci. 4: 19121923.

Pearson, K. G., and C. S. Goodman (1979) Correlation of variability in structure with variability in synaptic connections of an identified interneuron in locusts. J. Comp. Neurol. 184: 141-166.

Pearson, K. G., R. K. S. Wong, and C. R. Fourtner (1976) Connexions between hair plate afferents and motorneurones in the cockroach leg. J. Exp. Biol. 64: 251-266.

Sakaguchi, D. S., and R. K. Murphey (1983) The equilibrium detecting system of the cricket: Physiology and morphology of an identified interneuron. J. Comp. Physiol. 150: 141-152.

Schmidt, J. T., C. M. Cicerone, and S. S. Easter (1978) Expansion of the half retinal projection to the tectum in goldfish: An electrophysiological and anatomical study. J. Comp. Neurol. 177: 257-278.

Schneider, G. E. (1973) Early lesions of superior colliculus: Factors affecting the formation of abnormal retinal projections. Brain Behav. Evol. 8: 73-109.

Tieman, S. B. (1984) Effects of monocular deprivation on geniculocortical synapses in the cat. J. Comp. Neurol. 222: 166-176.

Wiesel, T. N., and D. H. Hubel (1965) Comparison of the effects of unilateral and bilateral eye closure on cortical unit responses in kittens. J. Neurophysiol. 28: 1029-1040.

Zucker, R. S. (1977) Synaptic plasticity at crayfish neuromuscular junctions. In Identified Neurons and Behavior of Arthropods, G. Hoyle, cd., pp. 49-66, Plenum, New York. 